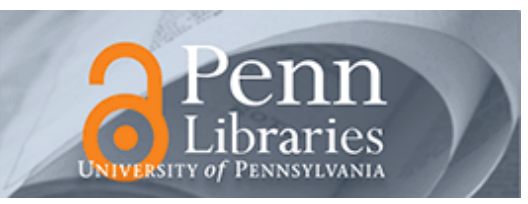

University of Pennsylvania ScholarlyCommons

\title{
$5-2012$
}

\section{Finite Element Model Predictions of Intracranial Hemorrhage From Non-impact, Rapid Head Rotations in the Piglet}

\author{
Brittany Coats \\ University of Pennsylvania, bcoats@seas.upenn.edu \\ Stephanie A. Eucker \\ University of Pennsylvania, saeucker@mail.med.upenn.edu \\ Sarah Sullivan \\ University of Pennsylvania \\ Susan S. Margulies \\ University of Pennsylvania, margulies@seas.upenn.edu
}

Follow this and additional works at: https://repository.upenn.edu/be_papers

Part of the Biomedical Engineering and Bioengineering Commons

\section{Recommended Citation}

Coats, B., Eucker, S. A., Sullivan, S., \& Margulies, S. S. (2012). Finite Element Model Predictions of Intracranial Hemorrhage From Non-impact, Rapid Head Rotations in the Piglet. International Journal of Developmental Neuroscience, 30 (3), 191-200. http://dx.doi.org/10.1016/j.ijdevneu.2011.12.009 


\title{
Finite Element Model Predictions of Intracranial Hemorrhage From Non-impact, Rapid Head Rotations in the Piglet
}

\author{
Abstract \\ Clinicians are charged with the significant task of distinguishing between accidental and inflicted head \\ trauma. Oftentimes this distinction is straightforward, but many times probabilities of injuries from \\ accidental scenarios are unknown making the differential diagnosis difficult. For example, it is unknown \\ whether intracranial hemorrhage $(\mathrm{IH})$ can occur at a location other than a focal contact site following a \\ low height fall. To create a foundation for predicting regional IH in infants, we sought to identify the \\ biomechanical response and injury threshold best able to predict IH in 3-5 day old piglets. First, finite \\ element (FE) model simulations of in situ animal studies were performed to ascertain the optimal \\ representation of the pia-arachnoid complex, cerebrospinal fluid and cortical vasculature (PCC) for \\ predicting brain strain and brain/skull displacement. Second, rapid head rotations resulting in various \\ degrees of $\mathrm{IH}$ were simulated $(n=24)$ to determine the biomechanical predictor and injury threshold most \\ closely correlated with IH. FE models representing the PCC with either spring connectors or solid \\ elements between the brain and skull resulted in peak brain strain and brain/skull displacement similar to \\ measured values in situ. However, when predicting $\mathrm{IH}$, the spring connector representation of the PCC had \\ the best predictive capability for $\mathrm{IH}$ with a sensitivity of $80 \%$ and a specificity of $85 \%$ when $\geq 1 \%$ of all \\ spring connectors had at least a peak strain of $0.31 \mathrm{~mm} / \mathrm{mm}$. These findings and reported methodology \\ will be used in the development of a human infant FE model to simulate real-world falls and identify injury \\ thresholds for predicting $\mathrm{IH}$ in infants.

\section{Keywords} \\ Subdural hemorrhage, Traumatic brain injury, Falls, Brain-skull displacement, Pediatric, Finite element

\section{Disciplines} \\ Biomedical Engineering and Bioengineering | Engineering
}




\title{
Finite Element Model Predictions of Intracranial Hemorrhage from Non-Impact, Rapid Head Rotations in the Piglet
}

\author{
Brittany Coats, $\mathrm{PhD}^{\mathrm{a}}$, Stephanie A. Eucker, MD PhD ${ }^{\mathrm{b}}$, Sarah Sullivan, MS ${ }^{\mathrm{c}}$, and Susan S. \\ Margulies, $\mathrm{PhD}^{\mathrm{C}}$ \\ ${ }^{a}$ Department of Mechanical Engineering, University of Utah, $50 \mathrm{~S}$. Central Campus Drive, Salt \\ Lake City, UT, 84112 USA. \\ bDepartment of Emergency Medicine, University of Virginia, PO Box 800669, Charlottesville, VA \\ 22908, USA. \\ 'Department of Bioengineering, University of Pennsylvania, 210 South $33^{\text {rd }}$ Street, Philadelphia, \\ PA 19104, USA.
}

\section{Abstract}

Clinicians are charged with the significant task of distinguishing between accidental and inflicted head trauma. Oftentimes this distinction is straightforward, but many times probabilities of injuries from accidental scenarios are unknown making the differential diagnosis difficult. For example, it is unknown whether intracranial hemorrhage (IH) can occur at a location other than a focal contact site following a low height fall. To create a foundation for predicting regional IH in infants, we sought to identify the biomechanical response and injury threshold best able to predict IH in 3-5 day old piglets. First, finite element (FE) model simulations of in situ animal studies were performed to ascertain the optimal representation of the pia-arachnoid complex, cerebrospinal fluid and cortical vasculature (PCC) for predicting brain strain and brain/skull displacement. Second, rapid head rotations resulting in various degrees of IH were simulated $(n=24)$ to determine the biomechanical predictor and injury threshold most closely correlated with IH. FE models representing the PCC with either spring connectors or solid elements between the brain and skull resulted in peak brain strain and brain/skull displacement similar to measured values in situ. However, when predicting IH, the spring connector representation of the PCC had the best predictive capability for IH with a sensitivity of $80 \%$ and a specificity of $85 \%$ when $\geq 1 \%$ of all spring connectors had at least a peak strain of $0.31 \mathrm{~mm} / \mathrm{mm}$. These findings and reported methodology will be used in the development of a human infant FE model to simulate real-world falls and identify injury thresholds for predicting $\mathrm{IH}$ in infants.

\section{Keywords}

subdural hemorrhage; traumatic brain injury; falls; brain-skull displacement; pediatric; finite element

\footnotetext{
(C) 2012 ISDN. Published by Elsevier Ltd. All rights reserved.

Corresponding Author: Brittany Coats, PhD, Mechanical Engineering, University of Utah, 50 S. Central Campus Drive, 2120 MEB, Salt Lake City, UT 84112, Telephone: (801) 585-0586, Fax: (801) 585-9826, brittany.coats@utah.edu.

Publisher's Disclaimer: This is a PDF file of an unedited manuscript that has been accepted for publication. As a service to our customers we are providing this early version of the manuscript. The manuscript will undergo copyediting, typesetting, and review of the resulting proof before it is published in its final citable form. Please note that during the production process errors may be discovered which could affect the content, and all legal disclaimers that apply to the journal pertain.
} 


\section{INTRODUCTION}

Traumatic brain injury (TBI) is one of the most common causes of death in childhood. The predominant etiologies of TBI in young children are motor vehicle crashes, falls, and abuse (Langlois et al. 2006). Clinicians are faced with the difficult challenge of identifying inflicted trauma in children. In some cases, primarily those involving severe head injury with no history of trauma or additional injury to extremities and limbs, this diagnosis is straightforward. However, in cases of minor head injury such as a linear skull fracture with an underlying and/or remote subdural hematoma, the differential diagnosis between inflicted and accidental trauma is more difficult. These diagnoses could be made easier if more data existed to identify regional probabilities of head injury from accidental scenarios.

One of the most common accidents in children is a low height fall. Head injury sustained from a fall is typically the result of two mechanisms. The first is the focal contact to the head that can result in scalp contusions, skull fracture, and intracranial hemorrhage underlying the impact site. The second is the rotation and translation of the head. If the head rotational acceleration is large enough, injuries such as axonal injury or intracranial hemorrhage (IH) may occur in locations other than the impact site. To date, however, it is unknown what fall scenarios result in accelerations that are large enough to produce these rotational, nonimpact injuries.

Predicting injuries from head impact or head rotation from a fall is challenging because the impact force and angular acceleration are dependent on the fall height, the floor surface, the direction of impact force to the skull (e.g., parietal impact vs occipital impact), and the age of the child (Bertocci et al. 2004; Coats and Margulies 2008). Epidemiology studies have tried to identify probabilities of injury from case studies of childhood falls, but small samples sizes and large variability in fall heights, surfaces, and child ages have resulted in broad conclusions that cannot be used to evaluate the probability of injury in a single fall (Smith et al. 1975; Nimityongskul and Anderson 1987; Musemeche et al. 1991; Root 1992; Reiber 1993; Tarantino et al. 1999; Macgregor 2000; Sawyer et al. 2000; Wang et al. 2001; Hennrikus et al. 2003; Haney et al. 2010).

Finite element (FE) modeling is a tool that can incorporate fall variables associated with a single fall and estimate regional deformations experienced by the brain and skull upon head impact and during head rotation. However, to correctly simulate fall scenarios, the brain and skull must be accurately represented in the FE model. This includes accurate material properties to define the deformation when a load is applied and accurate interactions between the brain and skull during focal contact and head rotation. The material properties of cortex, white matter, skull and suture have been measured, but the properties of the piaarachnoid complex with cerebrospinal fluid and cortical vasculature (PCC) are not well documented. Additionally, a unified approach to representing the complex structure in FE models has not been established. (Ruan et al. 1994; Kuijpers et al. 1995; Couper and Albermani 2008; Roth et al. 2008; Chafi et al. 2009; Yan and Pangestu 2011). The representation of the PCC in FE models has been found to significantly affect predictions of $\mathrm{IH}$ in adult animals undergoing non-impact head rotations (Takhounts et al. 2008). This finding is reasonable considering the deformation of the brain and the relative motion of the brain to the skull are recognized causes of subdural and subarachnoid hemorrhage (Depreitere et al. 2006), and will be influenced by the properties associated with the PCC.

To gain better insight into how to represent the PCC in a human infant FE model, we developed a FE model of a 3-5 day old piglet head and simulated several in situ and in vivo animal experiments. The objectives were to 1) identify the best representation of the PCC in an FEM for predicting IH from head rotation, and 2) identify the best biomechanical 
predictor and injury threshold for $\mathrm{IH}$ in piglets undergoing head rotation. To accomplish these objectives, we developed a multifaceted approach (Fig. 1). First, a partial 3-5 day old piglet FEM was created to compare predictions of brain strain and brain/skull displacement during head rotation to measured values in a previously published study (Ibrahim et al. 2010). The representation of the PCC that resulted in the closest approximations to the real brain strains and brain/skull displacements was then implemented into a full 3-5 day old piglet FEM. This FEM was used to simulate 6 experiments in which an animal's head was rotated in the axial direction. An ROC analysis was performed to determine the best biomechanical predictor of IH in the animal experiments. This predictor was then tested against additional simulations of 18 animal experiments in which the animal's head was rotated in either the axial, sagittal or coronal directions. Data from this study will provide insight into how to represent the PCC in a human infant FE model and what biomechanical predictors best correlate to IH during head rotation from a low height fall. Injury thresholds for predicting IH in the human infant FE model will be identified in another study by comparing IH predictions to real-world cases of falls in children.

\section{MATERIALS and METHODS}

\subsection{FE Model Development}

Geometry for the 3-5 day old piglet brain and skull was created by digitizing consecutive coronal computed tomography (CT) images of a 4 week old pig brain and brainstem $(512 \times$ 512 pixel, $1 \mathrm{~mm}$ thick, $F O V=15 \mathrm{~cm}$ ). A three-dimensional representation was configured from the digitized points (MIMICS 9.0, Materialize, MI) and then scaled to the dimensions of a 3-5 day old piglet brain. To identify scaling factors, measurements of the anteriorposterior, lateral, and superior-inferior lengths of the cerebrum were calculated from color photographs of ten 3-5 day old and ten 4-week old fixed piglet brains. The average length of each direction was used to determine the scale factor. Standard deviations of measurements within each group were smaller than differences between the groups. The scaling factor in each direction ranged from 0.88 to 0.91 . Because there were only a slight difference in the directional scale factors, 0.89 was used as the scale factor for all three dimensions.

The inner table of the skull was created by extending the cortical surface of the brain outward $1 \mathrm{~mm}$ and smoothing the surface to eliminate the gyral pattern (Fig. 2). Using calipers, the height and width of a 3-5 day old piglet falx were measured in vivo and ex vivo, and used to establish the geometry of the falx in the FE model.

To identify an appropriate representation of the pia-arachnoid complex with cerebrospinal fluid and cortical vasculature (PCC), 5 contact conditions between the brain and skull were implemented separately, and results were compared to experimentally obtained validation data (See 2.2 FE Model Validation). For the first contact condition, the surface nodes of the brain were tied to the skull so that the brain surface displaced with the skull. For the second contact condition, the brain was allowed to move relative to the skull but a coefficient of friction of 0.2 (Miller et al. 1999) dictated the contact interaction. For the third contact condition, the brain was free to move relative to the skull and coefficient of friction was set to 0 (pure slip). These 3 conditions represent the spectrum of resistance that the PCC may provide to the relative sliding motion of the brain along the skull. In a fourth contact condition, every surface node of the brain was connected to the skull using two-dimensional spring connector elements. These connectors were created to mimic the distensible tethering of the cortical vasculature in the PCC. The elastic modulus, cross-sectional area and length of cortical veins from autopsy (Monson et al. 2005) were used to define the stiffness of these connectors. Lastly, the fifth interaction represented the PCC with solid hexahedral elements with cerebrospinal fluid (CSF) properties. The volumetric response of the elements was 
determined using Mie-Gruneisen equations of state with a linear relationship between pressure and specific energy as described in Equation [1] (Meyers 1994).

$$
p=p_{H}\left(1-\frac{\Gamma_{0} \chi}{2}\right)+\Gamma_{0} \rho_{0} E_{m}
$$

In this equation, $\Gamma_{0}$ is a material constant, $\mathrm{E}_{\mathrm{m}}$ is the internal energy per unit mass, $\rho_{0}$ is the density of the material, and $\mathrm{p}_{\mathrm{H}}$ is the Hugoinot pressure and is defined by Equation [2] when assuming linear pressure-energy relationship. The volumetric compressive strain, $\chi$, is defined by the change in volume as indicated in Equation [3].

$$
\begin{gathered}
\mathrm{p}_{\mathrm{H}}=\frac{\rho_{0} \mathrm{c}^{2} \chi}{(1-\mathrm{s} \chi)^{2}} \\
\chi=1-\frac{V}{V_{0}}
\end{gathered}
$$

In situations of small strain, $\rho_{0} \mathrm{c}^{2}$ is equivalent to the bulk modulus, $\mathrm{K}$. For these simulations, the particle velocity coefficient, $\mathrm{s}$, and the material constant, $\Gamma_{0}$, was assumed to be zero. This resulted in a volume $(V)$ - pressure $(P)$ relationship driven by the bulk modulus $(K)$ of CSF as illustrated in Equation [4]. Because CSF is a viscous Newtonian fluid (Bloomfield et al. 1998), the shear stress $(\tau)$ of the elements was linearly related to the strain rate $(\dot{\gamma})$ and viscosity $(\eta)$ of the material as described in Equation [5]. As a layer sandwiched between brain and skull, PCC solid elements were tied to the brain and to the skull. During axial simulations, the compliant properties of the CSF caused excessive distortion in the brain. Therefore, elastic spring elements with the properties of bridging veins (Monson et al. 2005) were added to tie the falx to the brain in the FE model with CSF representing the PCC. In all other boundary condition representations, the falx was tied to the surface of the skull, and the interaction between the brain and the falx was defined to be the same as the interaction between the brain and skull.

$$
\begin{gathered}
P=-K \frac{\Delta V}{V_{0}} \\
\tau=2 \eta \dot{\gamma}
\end{gathered}
$$

The brain and brainstem were characterized as non-linear homogenous, isotropic hyperelastic and viscoelastic materials using a first-order Ogden strain energy equation to define the stress-strain response. Derivation and validation of this equation has been previously published by Prange and Margulies (2002). The falx was assumed to be an isotropic, elastic material. The elastic modulus of adult human dura has been reported by Galford and McElhaney (1970), but only stiffness has been reported for fetal dura (Bylski et al. 1986). To obtain an elastic modulus for the pediatric dura used in the FEM, the adult elastic modulus was converted to a stiffness $(\mathrm{N} / \mathrm{m})$ based on the average dimensions of the specimens. This resulted in a value approximately 2 times greater than the stiffness reported for fetal dura mater. Assuming that the ratio is the same for elastic moduli, the adult value reported by Galford and McElhaney (31.7 MPa) was halved to estimate the pediatric dura modulus. A rigid shell was used to represent the skull. All material properties and constants are reported in Table 1. 
A convergence study on the brain mesh was performed to investigate the stability and determine the optimum mesh density of the FE model while minimizing run time. Convergence was verified for each region, each output variable, and each head rotation direction simulated (sagittal, axial, coronal). To perform a convergence study with the FE model representing the PCC with connectors, we adjusted the elastic strength of the connector with each mesh density, to ensure the overall constraint of the brain was the same. The FE model resulting from the findings of the convergence study was composed of 17,587 elements (13,018 brain and brainstem hexahedral elements, 1,891 falx tetrahedral elements, and 2,678 skull rigid elements) plus 2,678 solid hexahedral elements for the fifth interaction investigated. All meshing was performed in Patran (MSC Software, Santa Ana, CA) for analysis in Abaqus Explicit (Simulia, Providence, RI).

\subsection{FE Model Validation}

To validate the material property assumptions and contact interactions of the FE model, we compared brain strain and brain/skull displacement predicted by the model to measured brain strain and brain/skull displacement data published for 3-5 day old piglets (Ibrahim et al. 2010). In the animal study, intact heads of 3-5 day old piglets were transected in a horizontal plane just superior to the orbits and lateral ventricles. The inferior portion of the transected head was embedded inside a custom-made cylindrical canister using PMMA. India ink dots were placed on the cut surface of the brain $(n=35-45)$ and skull/PMMA ( $n=6)$. A plexiglass plate was placed on top of the transection leaving approximately $1 \mathrm{~mm}$ of space between the brain and the plate. This gap was filled with a clear water-based lubricant to ensure a frictionless boundary condition. Silicone caulk was applied to the border of the plexiglass plate to seal it against the skull. Canisters were rotated $65^{\circ}$ at $50 \mathrm{rad} / \mathrm{s}$ while a high speed ( $2500 \mathrm{fps}$ ) digital camera recorded the motion. Completion of experiments occurred within 5 hours post-mortem. An in-house analysis program in MATLAB (MathWorks, Natick, MA) tracked the dots and calculated brain tissue strains and brainskull displacements. A total of three hemisection canisters were created, each from a separate animal.

To replicate the setup of the hemisection studies in the FE simulations, a horizontal brain transection was made in the three-dimensional FE model geometry and a rigid plate with a pure slip boundary condition was placed $1 \mathrm{~mm}$ above the cut surface with the exterior plate nodes tied to the skull nodes. This resulted in a three-dimensional model that mimicked the setup of the experimental studies. The velocity time history measured in each hemisection experiment was used as the load input for the simulations. Each of the 5 contact conditions used to represent the PCC were incorporated into a FEM and used to simulate the hemisection experiments.

Every ink dot on the brain in the hemisection study was matched to a corresponding node in the FE model. If an ink dot did not correspond to the exact location of a node, several surrounding nodes were selected such that their coordinates would average to the approximate location of the ink dot. The peak principal strain across all time points was extracted for each selected node. If multiple nodes represented the location of an ink dot, the principal strains for those nodes were averaged for each time point and the peak across all time points extracted. Strain distribution plots were created for both the FE model and experimental hemisection brain strains. A Kolmogorov-Smirnov goodness-of-fit test for continuous distributions was used to determine if the population distributions of peak principal strain were significantly different between the FE model and the experimental hemisection studies. A p-value $<0.05$ indicated that there was a significant difference in distributions and that the FE model was not a good representation of the experimental data. 
If the distributions were not significantly different, the cut surfaces of the brain in the FE model and in the hemisection canister were divided into four regions (anterior left, anterior right, posterior left, posterior right). Strain distributions in each region were plotted and the Kolmogorov-Smirnov tests were repeated to identify if the FE model was able to predict regional principal strain.

For brain-skull displacement analysis in the experimental hemisection studies, 4 ink dots around the skull were paired with the 3 closest ink dots on the brain surface $(\sim 2-3 \mathrm{~mm}$ inward from the cortical surface of the brain). Coordinates of all 12 skull-brain dot pairs were tracked using an in-house MATLAB program, and the largest magnitude displacement across time for each skull-brain pair was extracted. Skull and brain dots were correlated with FE model nodes and the largest magnitude displacement across time for each skull-brain pair in the FE model was extracted. For each contact condition, brain/skull displacements in the experimental hemisection studies and FE model (anterior, posterior, left, right) were plotted against each other and a linear regression was used to assess the validity of the FE model prediction of brain/skull displacement. A significant linear regression $(p<0.05)$ with the $95 \%$ confidence limits of the slope and y-intercept containing one and zero, respectively, was considered a good prediction of brain/skull displacement.

\subsection{Hemorrhage Prediction}

After the FE model was validated for its predictions of brain-skull displacement and brain strain, we simulated previously published animal experiments (Eucker 2009) to identify the biomechanical response that best predicts intracranial hemorrhage, to establish an appropriate injury threshold for that predictor, and to assess the sensitivity and specificity of our predictions using that predictor and threshold. In these animal experiments anesthetized 3-5 day old piglets were subjected to a rapid, inertial (non-impact) head rotation in the sagittal $(n=3)$, coronal $(n=6)$, or axial $(n=14)$ planes. Angular accelerations ranged from 26$85 \mathrm{krad} / \mathrm{s}^{2}$ and angular velocities ranged from 130-220 rad/s, producing a spectrum of traumatic brain injury from mild (little or no neuropathology) to severe (diffuse axonal injury with bilateral subdural and subarachnoid hemorrhage). Animals were euthanized and perfusion fixed 6 hours after injury. For each animal, the top of the skull was carefully removed and digital photographs were taken of the brain with overlying dura. The brain was then removed from the cranium and 'injury day' digital photographs were taken of the superior, inferior, left and right surfaces of the intact brain. Brains were placed in 10\% formalin for at least 5 days and then removed so that 'post-formalin' photographs could be taken.

Using anatomical markers in the post-formalin photographs, the brain surface was evaluated for five regions (anterior left, anterior right, posterior left, posterior right, and midline) and the percent of brain surface covered by hemorrhage in each region was calculated by counting the number of pixels containing blood and dividing by the total number of pixels in that region. This resulted in 5 regional intracranial hemorrhage percentile (IHP) scores for each brain. Care was taken to not count surfaces multiple times if they appeared in multiple views (Fig. 3). A total IHP score for each animal was calculated by summing pixels containing blood from all regions and dividing it by the sum of all pixels in every region. All images used in this study result in similar IHP scores if injury day photos are used instead of post-formalin photos.

For validation, intracranial hemorrhage was considered a binary variable. Several animal brains contained only a small amount of hemorrhage (total IHP: 3-15\%) and it was uncertain if the FE model could detect such subtleties or whether these small amounts would be relevant in a clinical setting using MR and CT imaging. Additionally, it is possible that blood did not originate in a region, but rather traveled from a neighboring region. Evaluation 
of the photographs indicated that blood spanning two regions was typically much greater in one region compared to the other and developing a criterion for defining blood as originating in a region would enhance the accuracy of the predictions. Thus, any region with an IHP score $\geq 25 \%$ was given the designation 'positive for hemorrhage' and anything less than $25 \%$ was designated as 'negative for hemorrhage.' This cut-off percentage was later evaluated to see if predictions were better or worse with higher and lower cutoff percentages.

Six animal experiments were selected to identify the biomechanical response that best predicted the presence or absence of hemorrhage, and to establish a threshold for the selected predictor. These six experiments represented a wide range of angular velocities $(144 \mathrm{rad} / \mathrm{s}-199 \mathrm{rad} / \mathrm{s})$ and total IHP scores $(1 \%-40 \%)$. For each simulation, the volume of the brain in the 3-5 day FE model was scaled uniformly according to the brain mass of the animal simulated and five biomechanical responses were investigated: cortical principal stress, cortical principal strain, cortical shear strain, brain/skull displacement, and connector strain (spring connector FE model only). The typical mechanism believed to cause intracranial hemorrhage is stretching of cortical and/or bridging vein vasculature during relative motion between the brain and the skull. For this reason, brain/skull displacement and connector strain were included as possible predictors. However, as cortical vessels enter the parenchyma of the brain they often bend or form junctions with other vessels. These changes in geometry may result in stress concentrations that lead to failure (Yamashima and Friede 1984). Therefore, cortical principal stress, cortical principal strain, and cortical shear strain were also included in the investigation. For each element in the FE model, peak values across all time points for each parameter were extracted. These peak values were assigned to one of 5 regions (anterior left, anterior right, posterior left, posterior right, midline) and population distributions of the peak values for each parameter were created in each region. The $1 \%, 10 \%$ and $50 \%$ values, defined as the largest strain or stress level experienced by at least $1 \%, 10 \%$, or $50 \%$ of the elements in each region, were extracted from each distribution (Fig. 4), resulting in 15 possible predictors. For each predictor, an in-house program developed in MATLAB incrementally swept through potential injury thresholds and calculated the sensitivity and specificity resulting from each threshold based on the presence or absence of IH in each region of the brain in each of the 6 animals. The sensitivity and specificity were plotted to create a receiver operating characteristic (ROC) statistical curve. An area of 0.5 under a ROC curve indicates that the predictor has no predictive capability and an area of 1.0 under a ROC curve indicates that the predictor has a perfect predictive capability. ROC curves for all predictors were examined, and the one resulting in the area under the curve closest to 1.0 was designated as the best predictor of intracranial hemorrhage. The threshold resulting in the highest specificity and sensitivity for the selected predictor was designated as the injury threshold. After a biomechanical predictor and injury threshold were identified, an additional 18 animal experiments were simulated and used to validate the FE model's ability to predict regional intracranial hemorrhage using this optimized threshold and the best PCC representation.

\section{RESULTS}

\subsection{Brain-Skull Interface}

Regardless of the contact condition used to represent the PCC, peak principal strains in the FE model were larger than those measured in the hemisection studies. To achieve similar strains, the shear modulus of the brain tissue was increased to 2.25 times the value measured in 3-5 day old piglets (Prange and Margulies 2002). This shear modulus (1,190 Pa) was used for all simulations, except for the simulation representing the PCC as solid elements, which had better correlation with brain strain when a shear modulus 1.5 times the reported value $(790 \mathrm{~Pa})$ was used. It was unknown which increase in brain stiffness $(2.25 \times$ or $1.5 \times)$ 
was closer to reality, so all representations of the PCC were evaluated equally with the stiffnesses stated above. As expected, the tied boundary condition between the brain and skull significantly $(\mathrm{p}<0.001)$ underestimated the overall brain strain in the hemisection studies while the frictional boundary condition significantly $(\mathrm{p}=0.0248)$ overestimated the strain (Fig. 5). The frictionless boundary condition (pure slip) resulted in unrealistic deformation of the brain tissue due to the separation of the brain from the skull, so this condition was removed from further evaluation. Both the spring connector and solid element interactions resulted in overall brain strain distributions that were not significantly different than those measured in Hemisection Study 1 ( $\mathrm{p}=0.29$ and $\mathrm{p}=0.49$, respectively, Fig. 5). Regional principal brain strain distribution was better for the spring connector interaction than for the solid element interaction (Fig. 6). The spring connector interaction resulted in no significant difference between strain distribution in the anterior left/right and posterior left regions, but the FE model predicted overall lower peak strains $(\mathrm{p}=0.01)$ in the posterior right. The solid element interaction resulted in no significant difference between strain distributions for the anterior right and posterior left regions, however, significantly underestimated strain in the anterior left region $(\mathrm{p}=0.032)$ and significantly overestimated strain in the posterior right region $(\mathrm{p}=0.014)$. Taken together, based on overall and regional brain strains, the spring connector representation of the PCC resulted in the best prediction of brain tissue deformation in the hemisection studies.

The brain-skull displacement from all four FE model representations of the PCC was linearly correlated with the displacement measured in Hemisection Study $1(\mathrm{p}<0.025$, Table $2)$. The frictional boundary condition had a poor coefficient of determination $\left(\mathrm{R}^{2}=0.43\right)$ and tended to overestimate brain-skull displacement by predicting peak displacements almost 2.5 times higher than the measured values (slope=2.37). The slopes of the linear regressions associated with tied, spring connector, and solid element interactions were closer to unity (slope $=0.843,0.935$ and 1.25 , respectively), but the coefficient of determination for the solid element interaction $\left(\mathrm{R}^{2}=0.63\right)$ was better than those for the tied $\left(\mathrm{R}^{2}=0.42\right)$ or spring connector $\left(\mathrm{R}^{2}=0.41\right)$ interactions. Based on these analyses, the solid element representation of the PCC was best at predicting brain-skull displacement in the hemisection studies.

The spring connector and solid element representations of the PCC were better at predicting brain strain and brain-skull displacement, respectively, so only these two FE models were used to simulation Hemisection Studies 2 and 3. These additional simulations reinforced the initial findings: the spring connector representation of the PCC remained slightly better at predicting brain strain and the solid element representation of the PCC remained slightly better at predicting brain-skull displacement. Because one representation of the PCC was not clearly better than the other, both the solid CSF and spring connector representations of the PCC were evaluated further to determine which representation of PCC had the highest sensitivity and specificity as a predictor of intracranial hemorrhages.

\subsection{Prediction of Intracranial Hemorrhage}

IHP scores in animals undergoing a rapid axial head rotation had increasing percentages of intracranial hemorrhage with increased angular head velocity (Fig. 7, Table 3), but coronal head rotations resulted in very little intracranial hemorrhage $(<5 \%)$ even at the highest angular velocity $(214 \mathrm{rad} / \mathrm{s})$. While the highest total IHP score for sagittal head rotations was only $35 \%$, there was a more uniform distribution of blood across all regions compared to axial and coronal head rotations. These trends in IHP score are similar to trends found from the pathology reports published previously (Eucker et al. 2011).

To identify the best biomechanical response (cortical principal stress, cortical principal strain, cortical shear strain, brain-skull displacement and connector strain) and injury threshold for predicting intracranial hemorrhage, 6 of 24 animal studies (starred in Table 3) 
were simulated using both of the FE models with the spring connector and solid element representations of the PCC. ROC curves were created for each biomechanical response. For the spring connector FE model, connector strain experienced by $50 \%$ of the connector elements resulted in the largest area under the curve ( 0.832 , Table 4$)$ but further evaluation of the ROC curve revealed that using strain experienced by $50 \%$ of the connector elements resulted in either a good sensitivity or a good specificity, but not both (Fig. 8). Using strain experienced by $1 \%$ of the connector elements, however, had a large area under the ROC curve (0.813) and resulted in a high sensitivity (86\%) and specificity (78\%) with a threshold of $0.31 \mathrm{~mm} / \mathrm{mm}$. Thus the strain experienced by $1 \%$ of the connector elements and a threshold of $0.31 \mathrm{~mm} / \mathrm{mm}$ were the selected biomechanical predictor and injury threshold, respectively, used for validation against the remaining 18 animals.

For the FE model using solid elements to represent the PCC, the best predictor of hemorrhage was the cortical principal stress experienced by $10 \%$ of the elements on the surface of the brain (area under ROC curve $=0.783$ ). A stress threshold of $45.4 \mathrm{kPa}$ resulted in a specificity and sensitivity of $83 \%$ and $71 \%$, respectively. This predictor and threshold were also selected to be validated against the 18 animals not used to identify the best predictor of $\mathrm{IH}$.

Following simulation the additional 18 animal studies, the $1 \%$ peak connector strain and $10 \%$ peak principal cortical stress were extracted from the spring connector and solid element FE models, respectively. The selected thresholds $(0.31 \mathrm{~mm} / \mathrm{mm}$ and $45.4 \mathrm{kPa}$ respectively) were used to predict the presence or absence of hemorrhage in each region of the brain. As anticipated from the ROC curves, predictions of hemorrhage from the elongation of spring connectors was better than that from brain surface principal stress in the solid element FE model (Table 5). Representing the PCC with spring connectors resulted in an overall sensitivity of $80 \%$ and specificity of $85 \%$ for predicting regional $\mathrm{IH}$, and representing the PCC with solid elements resulted in a sensitivity of $70 \%$ and a specificity of $77 \%$. Comparing the two representations of the PCC regionally, it is clear that the spring connector representation is better at predicting hemorrhage in the posterior left and midline regions compared to the solid element representation. For the remaining regions, the predictions were comparable between the two representations of the PCC. Overall, more accurate predictions of hemorrhage in the spring connector FE model occurred in the posterior left and midline regions and the least accurate prediction of hemorrhage occurred in the posterior right region of the FE model. This is partly due to the small number of brains with positive IH scores in the posterior right region.

\section{DISCUSSION}

Of the four brain-skull interactions investigated, using spring connectors or solid elements to represent the pia-arachnoid complex, cerebrospinal fluid and cortical vasculature resulted in the best correlation between calculated and measured brain strain and brain-skull displacement in simulations of in situ animal experiments. Interestingly, however, to achieve this similarity, brain stiffness had to be increased 2.25 or 1.5 times the reported in vitro shear modulus for 3-5 day old pigs. One reason this adjustment may have been necessary is because of the difference between material properties measured in vitro and those measured in situ. In a comparison of in situ and in vitro brain material properties of 4 week old pig, Gefen and Marguiles (2004) reported that both the short and long term shear moduli calculated from indentation experiments were 1.4 times stiffer when measured in situ compared to in vitro. They concluded that the encasement of the brain significantly affected the mechanical response of the tissue. Another possible factor contributing to the increased stiffness could include higher strain rates experienced in the FE models compared to material property tests. Nicolle et al (2005) report a 1.6 times increase in porcine corona 
radiata stiffness when testing frequencies are increased 10-fold. Similar increases in modulus with increased strain rate have been reported for adult bovine brain (Bilston et al. 1997), adult porcine gray matter (Arbogast and Margulies 1997; Brands et al. 1999) and adult human brain (Shuck and Advani 1972), emphasizing the importance of verifying material property selection for FE models.

Using spring connectors to represent the PCC resulted in a better match to brain strain measurements in two of the three hemisection studies compared to solid elements. However, using solid elements resulted in better predictions of brain-skull displacement in two of the three hemisection studies compared to spring connectors. Correlations of each model with only two out of three of the canister studies may be due to the structural integrity of the PCC in the third hemisection canister. In Ibrahim (2010), the hemisection canisters were either rotated multiple times at low velocities, or rotated a a single low velocity, a high velocity, and then another single low velocity. Measurements of brain strain were not significantly different in the repeated low velocities studies. However, measurements of brain strain were significantly different between the rotation of the canister at a low velocity following the high velocity rotation compared to the low velocity rotation prior to the high velocity rotation. This indicates that 1 ) there was some structural integrity of the PCC initially and 2) the high velocity rotations damaged that structural integrity. It is unknown if the structural integrity of the PCC in each hemisection canister was exactly the same as in vivo.

It is interesting that using spring connectors to represent the PCC resulted in a slightly better predictions of brain strain and using solid elements to represent the PCC resulted in slightly better predictions of brain-skull displacement. One important distinction between the two representations of the PCC is that one assume the CSF guides the response of the PCC to loading and the other assumes the cortical vasculature guides the response of the PCC to loading. In reality it is likely a mix of the two responses and further improvement to the PCC may be made by incorporating both the CSF and the cortical vasculature into a single representation of the PCC.

After comparing FE model predictions to experimental data from piglets undergoing a nonimpact rapid head rotation, it was determined that the best predictor of intracranial hemorrhage was the strain (percent change in length) experienced by $1 \%$ of the spring connector elements (Fig. 4). This finding is not surprising given that intracranial hemorrhage from non-impact head rotation is thought to occur by the stretching and ultimate failure of vessels as the brain moves relative to the skull. Absolute brain-skull displacement was not as good of a predictor as connector strain because the connectors were various lengths $(0.012$ $6.9 \mathrm{~mm}$ ), as is true of cortical vessels in animals and humans, and a small displacement may minimally affect a long vessel, but cause considerable damage to a small vessel.

The threshold of connector strain that best predicted intracranial hemorrhage in 3-5 day old pigs was $0.31 \mathrm{~mm} / \mathrm{mm}$ ( $31 \%$ percent change in length). There is no failure strain data reported for newborn porcine cortical veins, but a value can be estimated by using failure properties of adult human and porcine vasculature reported in the literature. The failure strain of adult human aorta and porcine aorta are $0.6 \mathrm{~mm} / \mathrm{mm}$ (Yamada 1970) and $0.8 \mathrm{~mm} /$ $\mathrm{mm}$ (Stemper et al. 2007), respectively, resulting in a human to pig species ratio of 0.75 . Applying this ratio to the average failure strain derived from human adult bridging veinsuperior sagittal sinus complexes $(0.248 \mathrm{~mm} / \mathrm{mm})$ results in a estimated adult porcine ultimate strain of $0.33 \mathrm{~mm} / \mathrm{mm}$ (Delye et al. 2006), similar to our newborn porcine threshold of $0.31 \mathrm{~mm} / \mathrm{mm}$. The stiffness of the connectors in this study was estimated from cortical vein properties and not bridging vein properties. Cortical vein failure is reported to be 1.15 times higher than bridging vein failure (Monson et al. 2005), resulting in an estimate of 0.38 $\mathrm{mm} / \mathrm{mm}$, still in the neighborhood of our threshold. One assumption in this estimation is that 
newborn vasculature is not significantly different than adult. While no significant effect of age has been reported for human bridging vein material properties among subjects 3-60 years old (Meaney 1991), it is unknown if this holds true for infants. More data is necessary to understand the alteration of cerebral vasculature material properties throughout the rapid developmental period of the infant (birth-3 years old). Nonetheless, the general agreement between our threshold and the estimated failure strain of adult porcine cortical veins supports our strategy to statistically compare FE model parameters to experimental data to obtain injury threshold information. A similar approach will be used to identify injury thresholds of IH in infants by simulating real world cases instead of experimental data.

The specificity and sensitivity of FE model predictions of intracranial hemorrhage were higher in the posterior left, anterior right, and midline regions of the brain. This may be attributed to the methodology used to create the IHP score. The FE model predicts intracranial hemorrhage immediately following a rapid head rotation, but IHP scoring was established from photographs taken 6 hours post-injury and may reflect the amount of blood created after the vessels have continued to bleed or track to other regions of the brain.

There were several assumptions made in the study that may affect the predictions of intracranial hemorrhage. First, the gyri in the FE model were eliminated, creating an idealized brain and skull surface. Cloots et al (2008) investigated the effect of heterogeneities on the cortical surface and reported an increase of 1.3-1.9 in maximum cortical stress for a variety of cortical heterogeneties compared to an idealized smooth cortical surface. It is unclear how the presence of gyri and sulci would alter the connector strain predictions of $\mathrm{IH}$, but it is anticipated that the presence of gyri and sulci would reduce the displacement and decrease the threshold determined in this study.

A cutoff percentage of $25 \%$ was used to define the presence or absence of intracranial hemorrhage. Given the small size of the 3-5 day old piglet brain, a $25 \%$ hemorrhage in a single region is about $1 \mathrm{~cm}$ in diameter. A cutoff percentage was used because the goal of the study was to create a model for detecting discernable blood on MR imaging. It ws felt that any amount smaller than this may not be identifiable in imaging. Additionally, it was desired to only predict blood in the regions where the blood originated. From the digital photgraphs of the animal studies, it was noticed that small amounts of blood leaked from one region to another. The application of a cutoff would eliminate this small amount of blood from the analysis and focus only on regions were blood originated. When identifying the best predictor and threshold using the initial set of 6 animals, this cutoff was varied and the sensitivity and specificity of predictions were evaluated. Decreasing the cutoff to $10 \%$ resulted in poor predictions of $\mathrm{IH}$ and increasing the cutoff to $30 \%$ resulted in similar predictions of IH. These ad hoc analyses indicate that the FE model would be unable to predict amounts of IH blood less than $1 \mathrm{~cm}$ in diameter.

In summary, predictions of intracranial hemorrhage in the 3-5 day old piglet were best made with a FE model containing spring connectors between the brain and skull to represent the pia-arachnoid complex with cerebrospinal fluid and cortical vasculature. The peak strain experienced by $1 \%$ of the connector elements resulted in the best predicitive attributes. The connector strain threshold that best predicted intracranial hemorrhage in the piglet was 0.31 $\mathrm{mm} / \mathrm{mm}$, which is reasonable based on reported ultimate strain in porcine and human bridging veins.

Clinicians are charged with the arduous task of distinguishing between accidental and inflicted trauma. Oftentimes the case is straightforward and the differential diagnosis is easy, but there exist numerous cases where the injuries are minor and there is not enough data to support or refute the plausability of the history provided. These cases will continue to be 
difficult to interpret until empirical evidence is gathered to aid in the diagnosis. Currently, development of a human infant FE model for predicting IH from low height falls is hindered by a lack of data on how best to represent the pia-arachnoid complex, cerebrospinal fluid and cortical vasculature. From the present study, we found spring connectors were the best representation of the PCC boundary and plan to incorporate this finding into a human infant FEM for simulating real-world cases of infant falls. We found the best predictor of IH was peak strain experienced by $1 \%$ of the spring connector elements. This biomechanical predictor will be calculated in the human infant FE model, compared to the presence of absence of regional IH as determined from MRI/CT images, and used to identify an appropriate injury threshold for predicting IH in infants. By using this multi-faceted approach, we can overcome the challenges in identifying pediatric specific injury thresholds and disseminate valuable data to clinicians and pediatric injury prevention researchers that will assist in the identification and prevention of traumatic brain injury in infants.

\section{Highlights}

- How the pia-arachnoid complex, cerebrospinal fluid and cerebral vasculature (PCC) are represented in FE models will significantly affect predictions of IH.

- Representing cortical vessel elasticity between the skull and brain resulted in the most accurate predictions of IH in piglets.

- Spring connector strain $\geq 0.31 \mathrm{~mm} / \mathrm{mm}$ was the most accurate injury threshold for predicting IH in immature pigs.

- Future human infant IH thresholds can be established by using human infant FE models to simulate real-world cases of trauma.

\section{Abbreviations}

$\begin{array}{ll}\text { CSF } & \text { Cerebrospinal fluid } \\ \text { FE } & \text { Finite element } \\ \text { IH } & \text { Intracranial hemorrhage } \\ \text { IHP } & \text { Intracranial hemorrhage percentile } \\ \text { PCC } & \text { Pia-arachnoid complex with cerebrospinal fluid and cortical vasculature } \\ \text { PMMA } & \text { Polymethylmethacrylic } \\ \text { ROC } & \text { Receiver operating characteristic } \\ \text { TBI } & \text { Traumatic brain injury }\end{array}$

\section{Acknowledgments}

The authors would like to thank Erica Hummel and Jill Ralston for their technical assistance with the IHP scoring. This study was made possible by the generous support of the CDC-NCIPC R01-CE001445, NIH-NINDS R01NS39679, and NIH-NINDS T32-NS043126-07.

\section{REFERENCES}

Arbogast, K.; Margulies, S. Regional differences in mechanical properties of the central nervous system; Proceedings of 41st Stapp Car Crash Conference, SAE; 1997.

Bertocci GE, Pierce MC, et al. Influence of fall height and impact surface on biomechanics of feet-first free falls in children. Injury. 2004; 35(4):417-424. [PubMed: 15037378] 
Bilston L, Liu Z, et al. Linear viscoelastic properties of bovine brain tissue in shear. Biorheology. 1997; 34(6):377-385. [PubMed: 9640354]

Bloomfield I, Johnston I, et al. Effects of proteins, blood cells, and glucose on the viscosity of cerebrospinal fluid. Pediatric Neurosurgery. 1998; 28:246-251. [PubMed: 9732257]

Brands, D.; Bovendeerd, P., et al. Comparison of the dynamic behaviour of brain tissue and two model materials; Proceedings of 43rd Stapp Car Crash Conference; San Diego, SAE. 1999.

Bylski DI, Kriewall TJ, et al. Mechanical behavior of fetal dura mater under large deformation biaxial tension. Journal of Biomechanics. 1986; 19(1):19-26. [PubMed: 3949813]

Chafi M, Dirisala V, et al. A finite element method parametric study of the dynamic response of the human brain with different cerebrospinal fluid constitutive properties. Proc Inst Mech Eng H. 2009; 223(8):1003-1019. [PubMed: 20092097]

Cloots R, Gervaise J, et al. Biomechanics of traumatic brain injury: influences of the morphologic heterogeneities of the cerbral cortex. Journal of Biomedical Engineering. 2008; 36(7):1203-1215.

Coats B, Margulies SS. Potential for head injuries in infants from low-height falls. J Neurosurg: Pediatrics. 2008; 2:1-10.

Couper Z, Albermani F. Infant brain subjected to oscillatory loading: material differentiation, properties, and interface conditions. Biomechanics and modeling in mechanobiology. 2008; 7:105-125. [PubMed: 17333090]

Delye H, Goffin J, et al. Biomechanical properties of the superior sagittal sinus-bridging vein complex. Stapp Car Crash Journal. 2006; 50:625-636. [PubMed: 17311180]

Depreitere B, van Lierde C, et al. Mechanics of acute subdural hematomas resulting from bridging vein rupture. J Neurosurg. 2006; 104:950-956. [PubMed: 16776340]

Eucker, S. Bioengineering. Philadelphia: University of Pennsylvania. PhD:; 2009. Effect of head rotation direction on closed head injury in neonatal piglets; p. 197

Eucker S, Smith C, et al. Physiological and histopathological responses following closed rotational head injury depend on direction of head motion. Experimental Neurology. 2011; 227(1):79-88. [PubMed: 20875409]

Galford J, McElhaney J. A viscoelastic study of scalp, brain, and dura. Journal of Biomechanics. 1970; 3(2):211-221. [PubMed: 5521539]

Gefen A, Margulies S. Are in vivo and in situ brain tissues mechanically similar? Journal of Biomechancs. 2004; 37(9):1339-1352.

Haney S, Starling S, et al. Characteristics of falls and risk of injury in children younger than 2 years old. Pediatric Emergency Care. 2010; 26(12):914-918. [PubMed: 21088634]

Hennrikus WL, Shaw BA, et al. Injuries when children reportedly fall from a bed or couch. Clin Orthop. 2003; (407):148-151. [PubMed: 12567141]

Ibrahim N, Natesh R, et al. In situ deformations int he immature brain during rapid rotations. J Biomech Eng. 2010; 132(4)

Kuijpers A, Claessens M, et al. The influence of different boundary conditions on teh response of the ehad to impact: a two-dimensional finite element study. Journal of Neurotrauma. 1995; 12(4):715724. [PubMed: 8683623]

Langlois, J.; Rutland-Brown, W., et al. Traumatic brain injury in the United States: emergency department visits, hospitalizations, and deaths. Atlanta (GA): Centers for Disease Control and Prevention, National Center for Injury Prevention and Control; 2006.

Macgregor DM. Injuries associated with falls from beds. Inj Prev. 2000; 6(4):291-292. [PubMed: 11144631]

Meaney, D. Bioengineering. University of Pennsylvania. Ph.D.; 1991. Biomechanics of acute subdural hematoma in the subhuman primate and man.

Meyers, M. Dynamic behavior of materials. New York: John Wiley \& Sons; 1994.

Miller, R.; Smith, D., et al. Comparing experimental data to traumatic brain injury finite element models; 43rd Stapp Car Crash Conference; San Diego, CA, SAE. 1999.

Monson KL, Goldsmith W, et al. Significance of source and size in the mechanical response of human cerebral blood vessels. Journal of Biomechanics. 2005; 35:737-744. [PubMed: 15713294] 
Musemeche CA, Barthel M, et al. Pediatric falls from heights. J Trauma. 1991; 31(10):1347-1349. [PubMed: 1942140]

Nicolle S, Lounis M, et al. Shear linear behavior of brain tissue over a large frequency range. Biorheology. 2005; 42:209-223. [PubMed: 15894820]

Nimityongskul P, Anderson LD. The likelihood of injuries when children fall out of bed. J Pediatr Orthop. 1987; 7(2):184-186. [PubMed: 3558802]

Prange M, Margulies S. Regional, directional, and age-dependent properties of brain undergoing large deformation. Journal of Biomechanical Engineering. 2002; 124:244-252. [PubMed: 12002135]

Reiber GD. Fatal falls in childhood. How far must children fall to sustain fatal head injury? Report of cases and review of the literature. American Journal of Forensic Medicine \& Pathology. 1993; 14(3):201-207. [PubMed: 8311050]

Root I. Head injuries from short distance falls. Am J Forensic Med Pathol. 1992; 13(1):85-87. [PubMed: 1585894]

Roth S, Raul JS, et al. Biofidelic child head FE model to simulate real world trauma. Computer Methods and Programs in Biomedicine. 2008; 90:262-274. [PubMed: 18342981]

Ruan JS, Khalil T, et al. Dynamic response of the human head to impact by three-dimensional finite element analysis. Journal of Biomechanical Engineering. 1994; 116(1):44-50. [PubMed: 8189713]

Sawyer JR, Flynn JM, et al. Fracture patterns in children and young adults who fall from significant heights. J Pediatr Orthop. 2000; 20(2):197-202. [PubMed: 10739282]

Shuck L, Advani S. Rheological response of human brain tissue in shear. Journal of Basic Engineering. 1972; 94:905-911.

Smith MD, Burrington JD, et al. Injuries in children sustained in free falls: an analysis of 66 cases. J Trauma. 1975; 15(11):987-991. [PubMed: 1195446]

Stemper B, Yoganandan N, et al. Biomechanical charcterization of internal layer subfailure in blunt arterial injury. Ann Biomed Eng. 2007; 35(2):285-291. [PubMed: 17151920]

Takhounts E, Ridella S, et al. Investigation of traumatic brain injuries using the next generation of simulated injury monitor (SIMon) finite element head model. Stapp Car Crash Journal. 2008 November.52:1-31. (2008). [PubMed: 19085156]

Tarantino CA, Dowd MD, et al. Short vertical falls in infants. Pediatr Emerg Care. 1999; 15(1):5-8. [PubMed: 10069302]

Wang MY, Kim KA, et al. Injuries from falls in the pediatric population: an analysis of 729 cases. J Pediatr Surg. 2001; 36(10):1528-1534. [PubMed: 11584402]

Yamada, H. Strength of Biological Materials. Baltimore: Williams and Wilkins Co.; 1970.

Yamashima T, Friede R. Why do bridging veins rupture into the virtual subdural space? Journal of Neurology. 1984; 47:121-127.

Yan W, Pangestu O. A modified human head model for the study of impact head injury. Computer methods in biomechanics and biomedical engineering. 2011 epubh ahead of print. 


\section{1) Identify best representation of PCC}

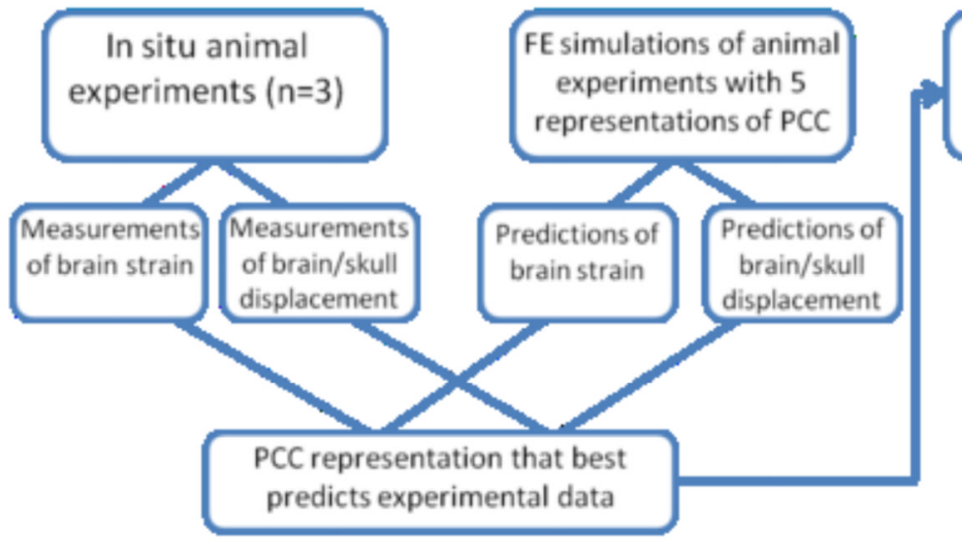

\section{2) Identify best predictor of $\mathrm{IH}$}

FE simulations of inertial animal experiments

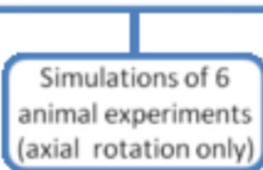
animal experiments (axial rotation only)

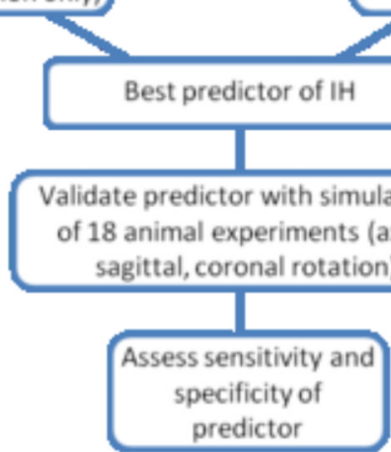

Fig. 1.

Multi-step approach used to identify the best representation of PCC and the best predictor of IH from head rotation. 


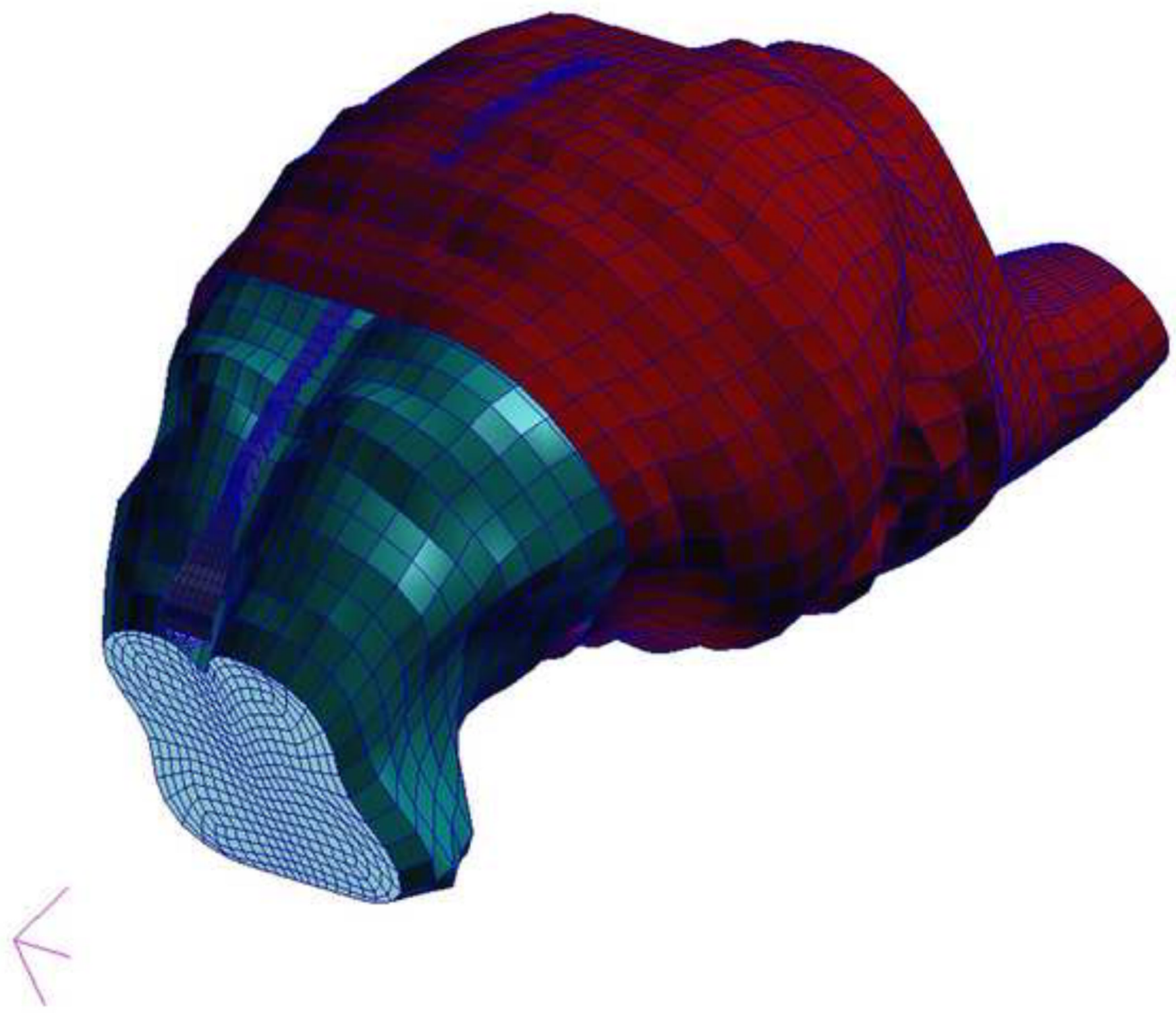

Fig. 2.

Finite element model of the 3-5 day old piglet brain and skull. Components include brain (green), brainstem, falx (blue), and skull (red). 

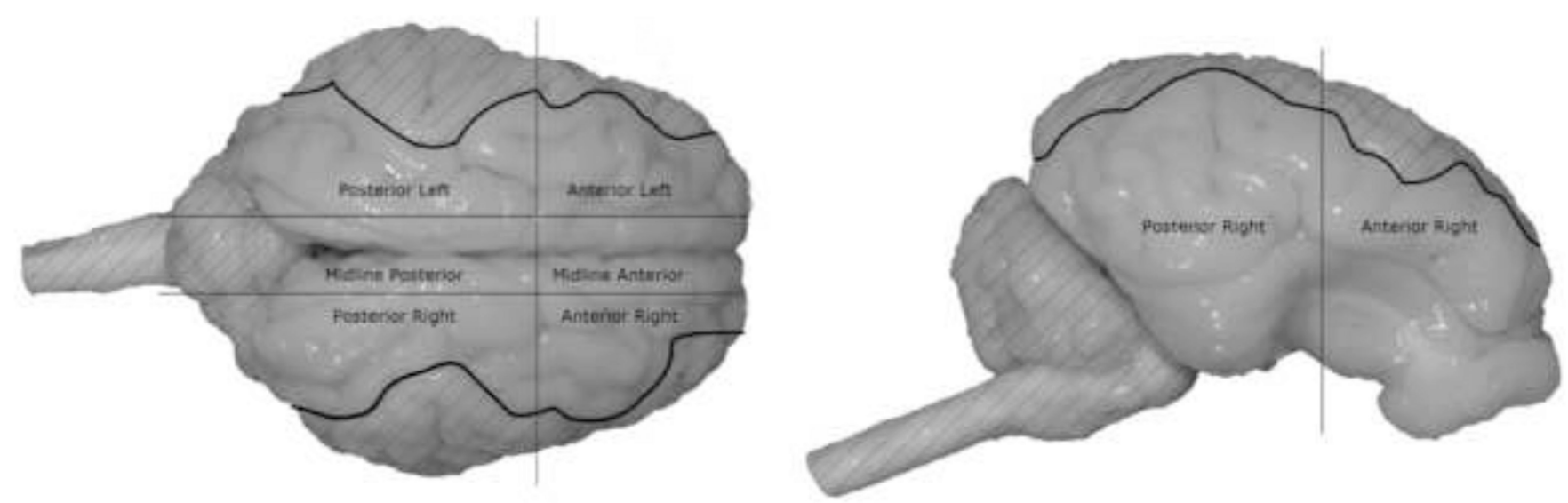

Fig. 3.

Using digital photographs, regional intracranial hemorrhage was quantified in each animal following a rapid head rotation. Using anatomical markers to divide the brain into 5 regions (anterior left, anterior right, posterior left, posterior right, and midline), the percent of brain surface covered by hemorrhage in each region was calculated by counting the number of pixels containing blood and dividing by the total number of pixels in that region. Hatched regions in the images indicate parts of the brain surface not quantified in that image view. This prevented double counting pixels present in multiple views. 


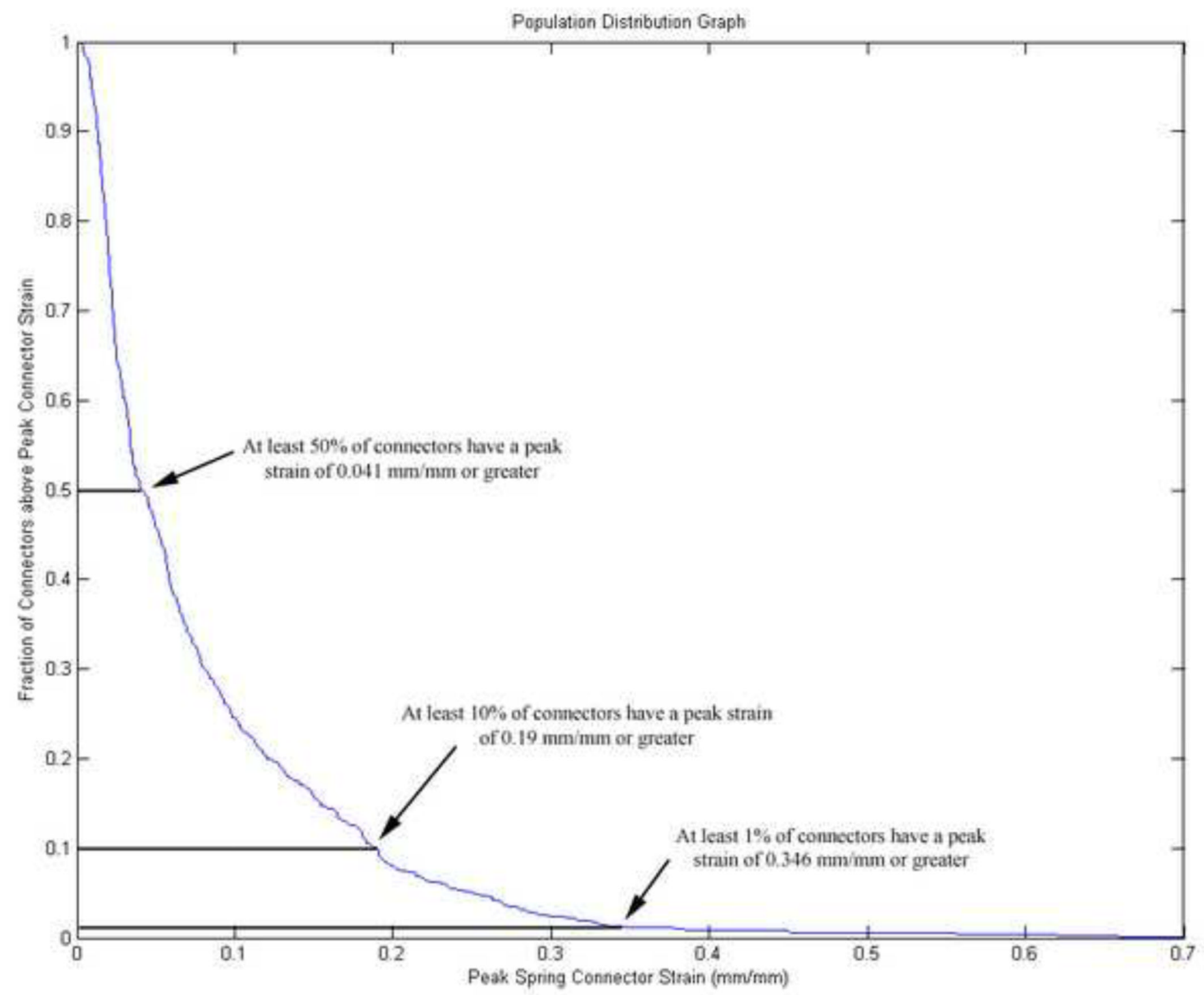

Fig. 4.

Example of a population distribution graph created from the FE model simulation data. Peak values for each biomechanical response (connector strain in this example) were extracted for each connector or element across all time points. The values that at least $1 \%, 10 \%$ and $50 \%$ of the connector or elements experienced were extracted to determine if they would be good predictors of IH in the piglets. 


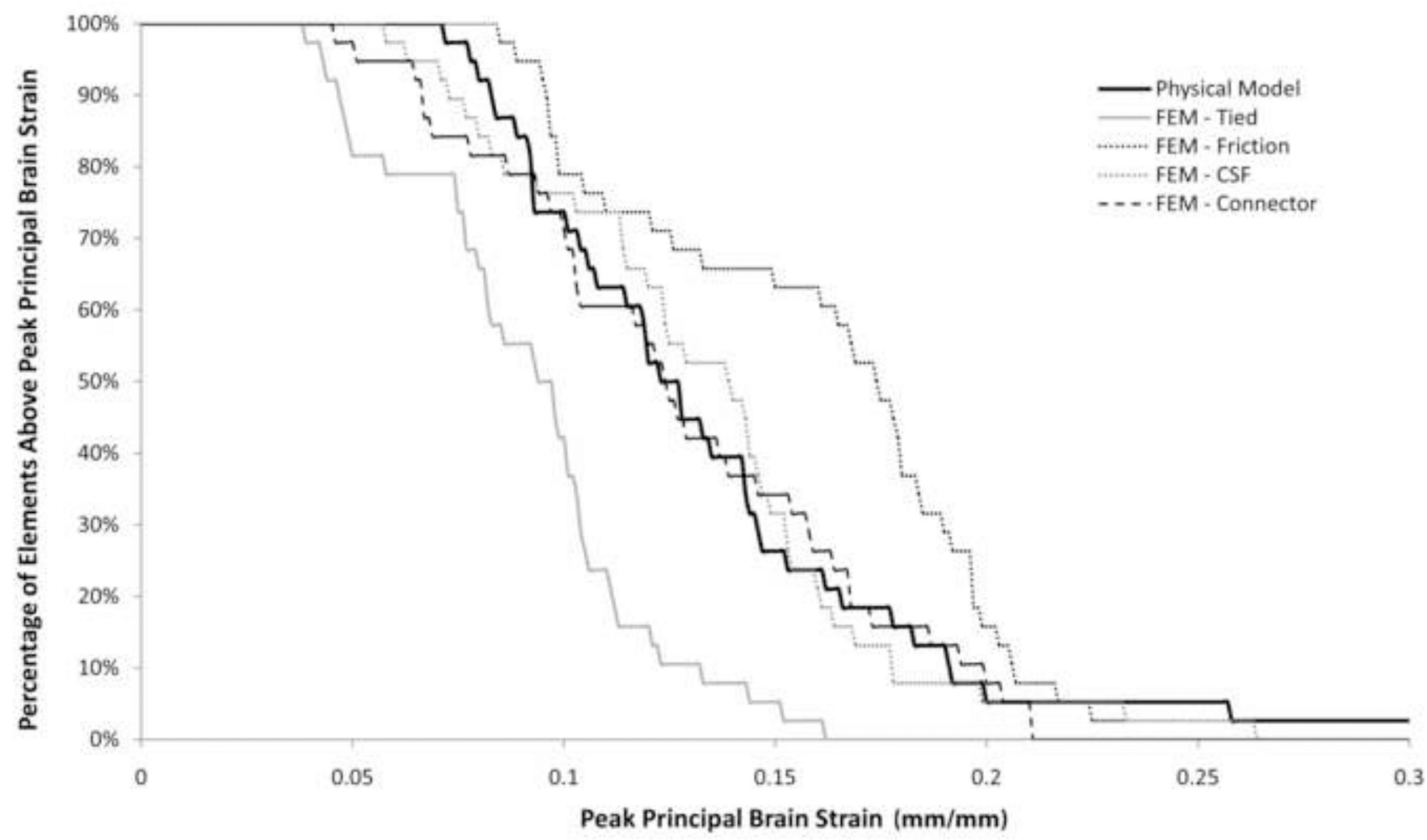

Fig. 5.

Comparison of brain strain distribution in Hemisection Study 1 (solid line) to FE model strain distributions using different models of PCC interactions. The solid element (lightdotted line) and spring connector (dark dashed line) models resulted in the best correlation to the experimental data. 


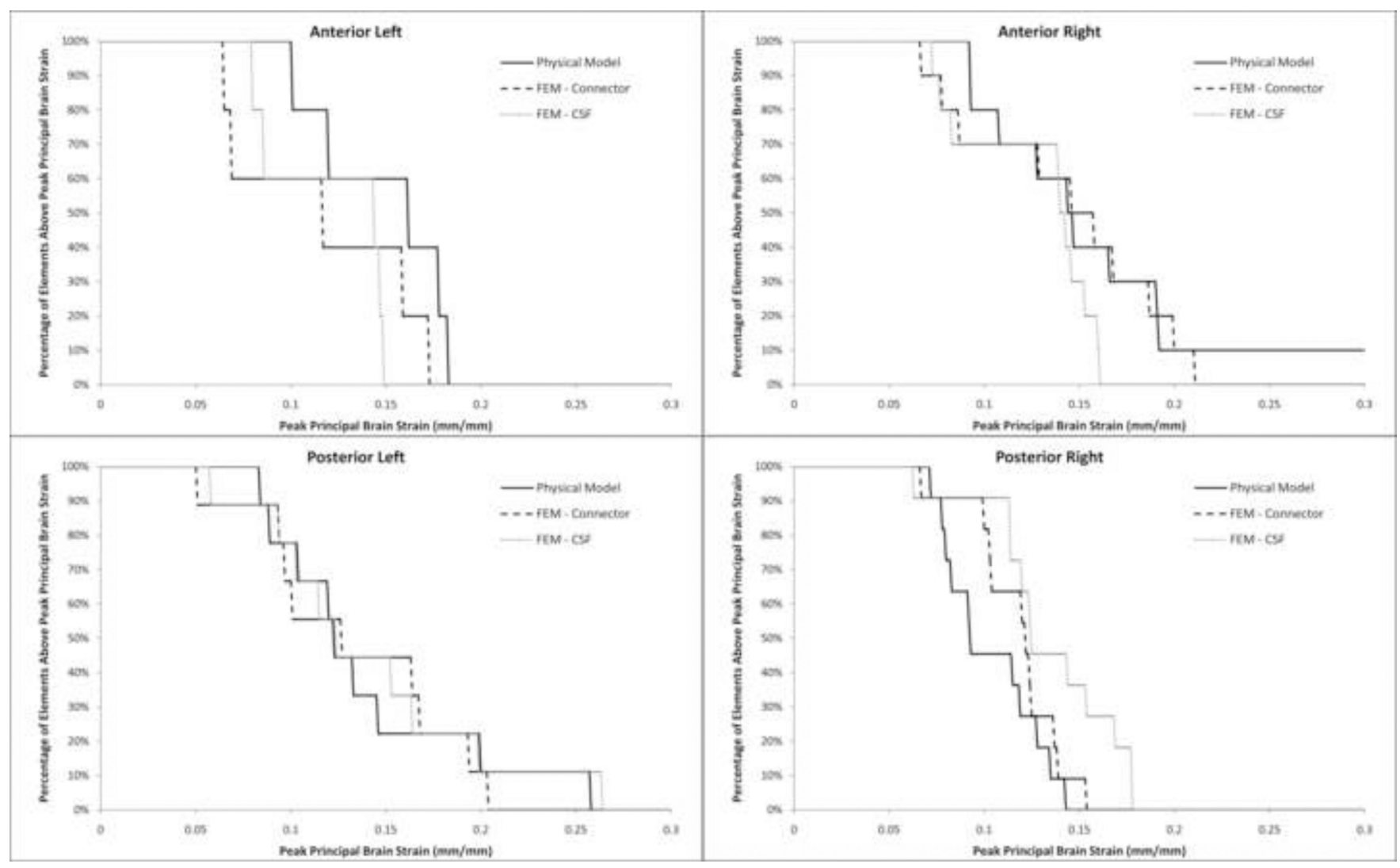

Fig. 6.

Comparison of regional strain distribution in Hemisection Model 1 (solid line) to the solid element and spring connector models. Better model correlation was found in the anterior right and posterior left regions of the brain. 


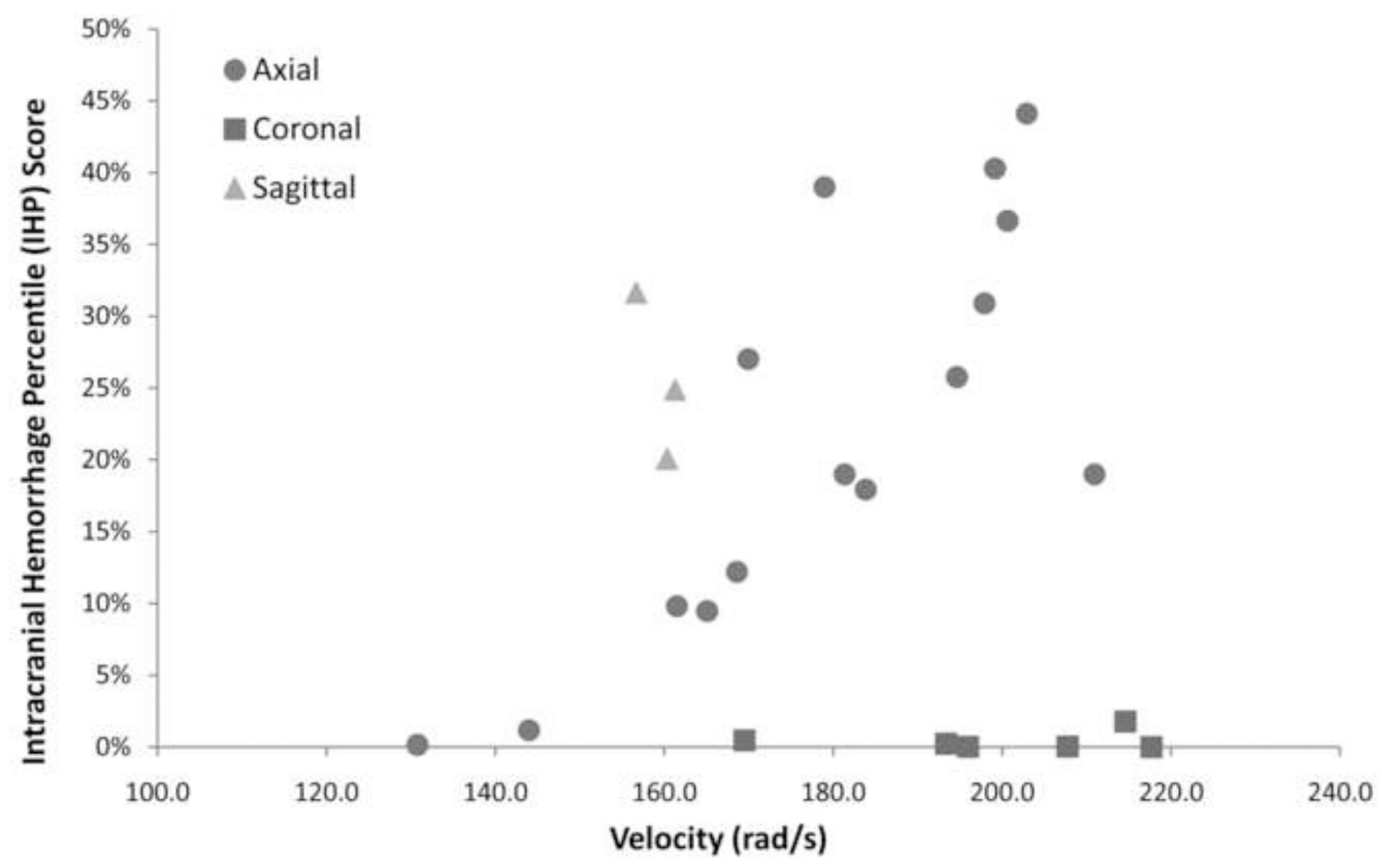

Fig. 7.

Intracranial hemorrhage percentile (IHP) score increased significantly with velocity for axial head rotations. Coronal head rotations resulted in overall low IHP scores even at high velocities $(214 \mathrm{rad} / \mathrm{s})$ and sagittal head rotations resulted in high IHP scores even at the lower velocities $(160 \mathrm{rad} / \mathrm{s})$. 


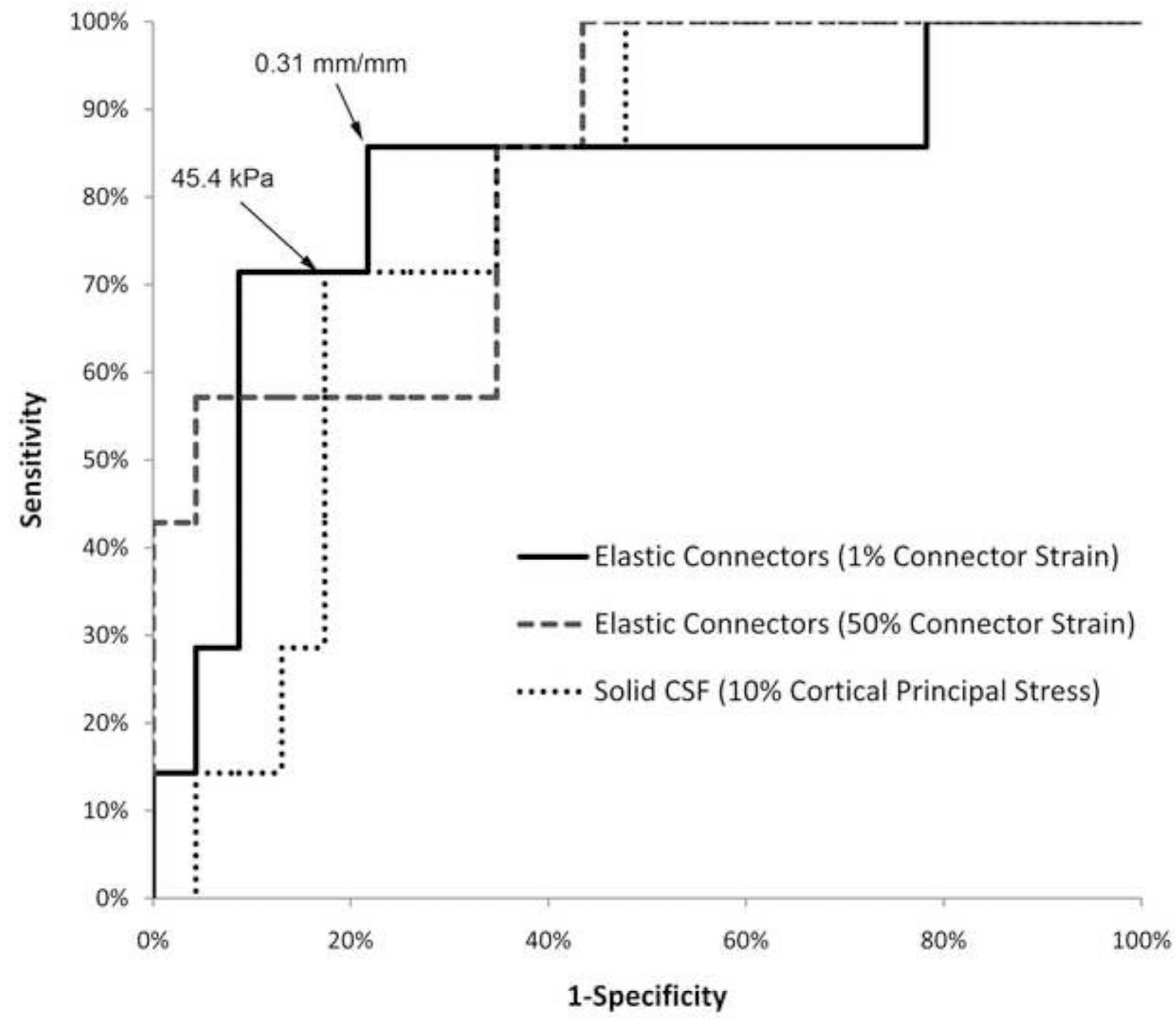

Fig. 8.

ROC curves for the three best biomechanical predictors from both the spring connector and solid element model. Strain experienced by $1 \%$ of connector elements in the spring connector model had the overall largest area under the ROC curve and resulted in the best prediction of intracranial hemorrhage using a threshold of $0.31 \mathrm{~mm} / \mathrm{mm}$. Cortical principal stress experienced by $10 \%$ of the brain elements was the best biomechanical predictor for the solid element model using a threshold of $45.5 \mathrm{kPa}$. 


\section{Table 1}

Material Properties used in the finite element model of a 3-5 day old piglet head.

\begin{tabular}{|c|c|c|}
\hline & Material Property & Source \\
\hline Brain/brainstem & $\begin{array}{c}\mu=526.9 \mathrm{~Pa} \\
\alpha=0.01 \\
C 1=0.332 \\
\mathrm{C} 2=0.389 \\
\tau 1=2.96 \mathrm{sec} \\
\tau 2=0.181 \mathrm{sec} \\
v=0.49999\end{array}$ & Prange \& Margulies (2002) \\
\hline Falx & $\begin{array}{c}\rho=1.13 \mathrm{~g} / \mathrm{cm}^{3} \\
\mathrm{E}=16 \mathrm{MPa} \\
v=0.45\end{array}$ & $\begin{array}{c}\text { estimated from data reported by } \\
\text { Galford \& McElhaney (1970) and } \\
\text { Bylski et al (1986) }\end{array}$ \\
\hline Connectors & $3460 \mathrm{~N} / \mathrm{m}$ & $\begin{array}{l}\text { estimated from data reported by } \\
\text { Monson et al. (2005) }\end{array}$ \\
\hline CSF & $\begin{array}{c}\rho=1.0 \mathrm{~g} / \mathrm{cm}^{3} \\
\mathrm{~K}=2.2 \mathrm{GPa} \\
\eta=0.727 \mathrm{mPa} \bullet \mathrm{s}\end{array}$ & Bloomfield et al. (1998) \\
\hline
\end{tabular}




\section{Table 2}

Summary of linear regression statistics comparing FE model predicted brain-skull displacement to measured brain-skull displacement in Hemisection Study 1.

\begin{tabular}{|rcccc|}
\hline & $\mathbf{R}^{\mathbf{2}}$ & $\begin{array}{c}\text { Slope } \\
\text { [95\% confidence } \\
\text { intervals] }\end{array}$ & $\begin{array}{c}\text { Y-Intercept } \\
\text { [95\% confidence } \\
\text { intervals] }\end{array}$ & $\begin{array}{c}\mathbf{p - ~} \\
\text { value }\end{array}$ \\
\hline Tied & 0.42 & $0.843[0.151,1.54]$ & $-0.254[-1.47,0.961]$ & 0.022 \\
Friction & 0.43 & $2.37[0.43,4.31]$ & $0.1738[-3.23,3.57]$ & 0.021 \\
Connectors & 0.41 & $0.935[0.146,1.72]$ & $0.065[-1.45,1.32]$ & 0.024 \\
Solid Element & 0.63 & $1.25[0.596,1.94]$ & $-0.286[-1.49,0.914]$ & 0.002 \\
\hline
\end{tabular}




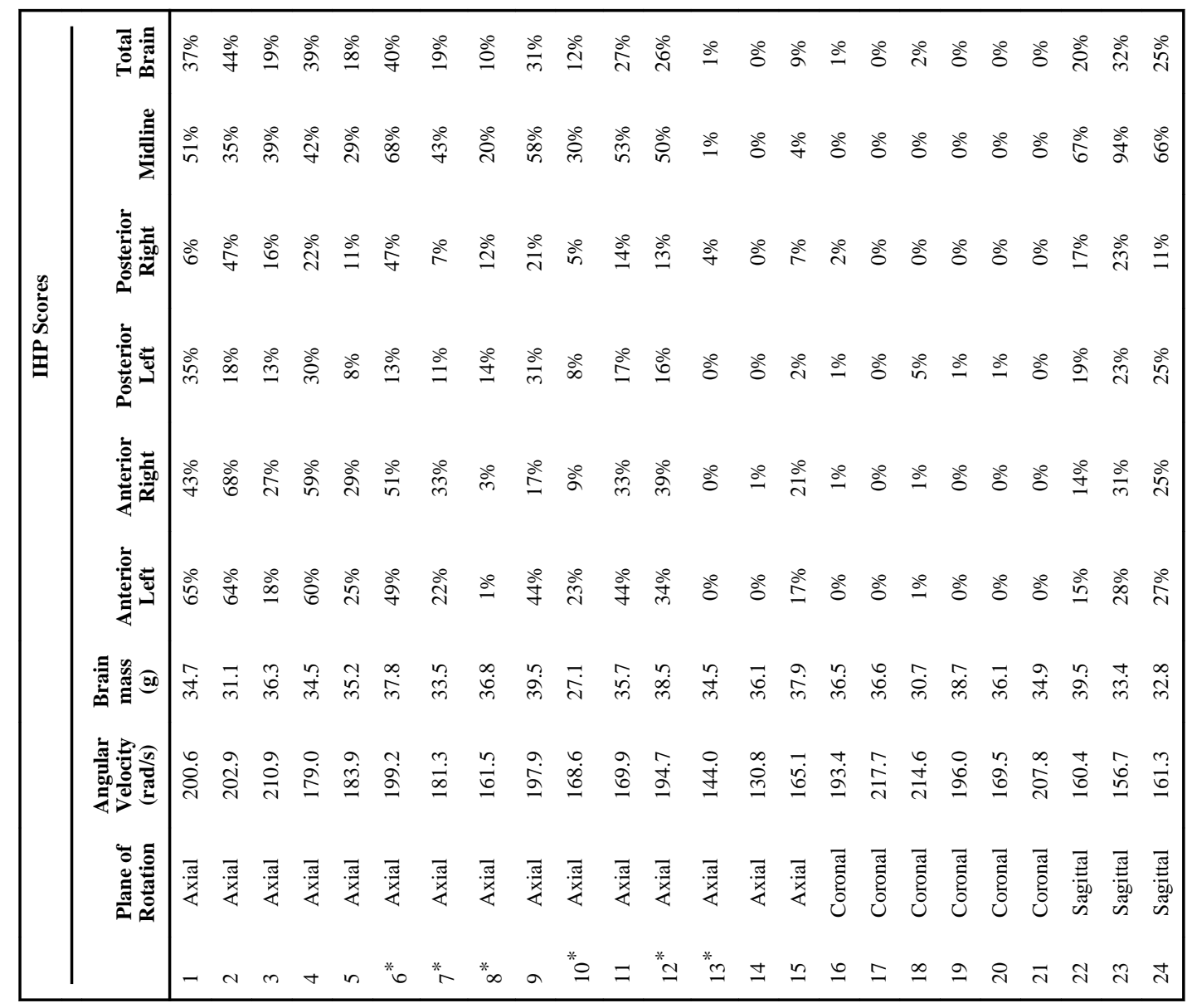


Int J Dev Neurosci. Author manuscript; available in PMC 2013 May 1. 


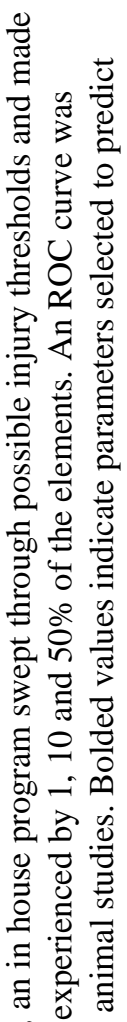

$\nabla+\overline{0}$

రิ

○芯

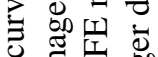

0 胥 I

○ 을

ช ฮ 을

झ

ষ) 00

으을

\begin{tabular}{|c|c|c|}
\hline & & 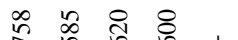 \\
\hline & in & $\hat{0}$ in \\
\hline & $\stackrel{\circ}{g}$ & 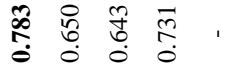 \\
\hline & $\stackrel{0}{2}$ & 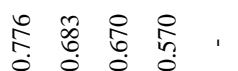 \\
\hline & $\stackrel{\circ}{\circ}$ & 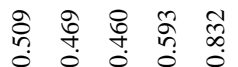 \\
\hline 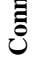 & $\stackrel{\circ}{\varrho}$ & 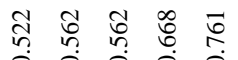 \\
\hline 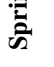 & $\stackrel{\circ}{\approx}$ & 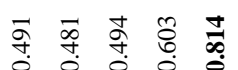 \\
\hline & & 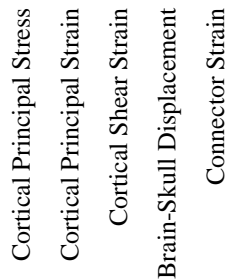 \\
\hline
\end{tabular}

Int J Dev Neurosci. Author manuscript; available in PMC 2013 May 1. 


\section{Table 5}

Sensitivity and specificity of the selected biomechanical predictors and thresholds when compared to IHP scores from the 18 animals not used in the development of the predictors.

\begin{tabular}{|rcccc|}
\hline & $\begin{array}{c}\text { Spring Connector FE Model: } \\
\text { 0.31 mm/mm > strain in 1\% } \\
\text { spring connector elements }\end{array}$ & $\begin{array}{c}\text { Solid Element FE Model: } \\
\mathbf{4 5 . 4} \\
\text { kPa > principal stress in 10\% } \\
\text { of surface brain elements }\end{array}$ \\
\hline & Sensitivity & Specificity & Sensitivity & Specificity \\
\hline Overall & $\mathbf{8 0 \%}$ & $\mathbf{8 5 \%}$ & $\mathbf{7 0 \%}$ & $\mathbf{7 7 \%}$ \\
\hline $\begin{array}{r}\text { Anterior } \\
\text { Left }\end{array}$ & $63 \%$ & $80 \%$ & $63 \%$ & $70 \%$ \\
$\begin{array}{r}\text { Anterior } \\
\text { Right }\end{array}$ & $80 \%$ & $88 \%$ & $100 \%$ & $70 \%$ \\
$\begin{array}{r}\text { Posterior } \\
\text { Left }\end{array}$ & $100 \%$ & $93 \%$ & $67 \%$ & $73 \%$ \\
$\begin{array}{r}\text { Posterior } \\
\text { Right }\end{array}$ & $0 \% *$ & $76 \%$ & $0 \%{ }^{*}$ & $71 \%$ \\
Midline & $90 \%$ & $100 \%$ & $60 \%$ & $88 \%$ \\
\hline
\end{tabular}

* Only 1 animal had an IHP score $\geq 25 \%$ in the posterior right region. Neither model predicted this single case and thus the sensitivity in this region was $0 \%$. 